\title{
Emergence of Novel Representations in Primary Motor Cortex and Premotor Neurons during Associative Learning
}

\author{
Neta Zach, ${ }^{1,2}$ Dorrit Inbar, ${ }^{1,2}$ Yael Grinvald, ${ }^{1}$ Hagai Bergman, ${ }^{1,2}$ and Eilon Vaadia ${ }^{1,2}$ \\ ${ }^{1}$ Department of Physiology, Hadassah Medical School, and ${ }^{2}$ The Interdisciplinary Center for Neural Computation, The Hebrew University of Jerusalem, \\ Jerusalem 91120, Israel
}

\begin{abstract}
Neurons in the motor areas of cortex play a key role in associating sensory instructions with movements. However, their ability to acquire and maintain representations of novel stimulus features, especially when these features are behaviorally relevant, remains unknown. We investigated neuronal changes in these areas during and after associative learning, by training monkeys on a novel reaching task that required associating target colors with movement directions. Before and after learning, the monkeys performed a well known center-out task. We found that during learning, up to $48 \%$ of the neurons developed learning-related responses, differentiating between the associative task and the center-out task, although movement kinematics were the same. After learning, on returning to the center-out task in which color was irrelevant, many of these neurons maintained their response to the associative task; they displayed novel sensitivity to the color of the target that was relevant during learning. These neuronal responses prevailed in both the primary motor cortex and the ventral and dorsal premotor cortices, without degrading the information that the neurons firing carried about movement direction. Our results show that motor cortical neurons can rapidly develop and maintain sensitivities to novel arbitrary sensory features such as color, when such features are behaviorally relevant.
\end{abstract}

Key words: motor learning; motor cortex; memory; association; single-unit activity; consolidation

\section{Introduction}

The primary motor cortex (M1) and the premotor areas are known to be involved in sensorimotor transformations and sensorimotor associations between spatial targets and the motor outputs required to achieve them (Kalaska et al., 1997; Georgopoulos, 2000). Efficient sensorimotor control also involves learning to adapt to novel environments. In this study, we examined the changes accompanying learning novel sensorimotor associations.

Many studies have reported involvement of these motor cortices in motor adaptation, learning, and consolidation of motor tasks after learning. The activation of M1 during learning has been shown using magnetic resonance imaging (MRI) (Karni et al., 1995, 1998) and mimicked by cortical microstimulation studies (Jackson et al., 2006). In addition, single neuron activity shows learning-related changes in the motor cortices (Mitz et al., 1991; Wise et al., 1998; Costa et al., 2004; Paz et al., 2005). However, we have little knowledge as to the extent of these changes; in particular, are learning-related responses in the motor cortices restricted to altering existing representations, or could novel parameters be represented as well? For example, in other primary

Received Nov. 5, 2007; revised Aug. 5, 2008; accepted Aug. 12, 2008.

This work was supported in part by the Bundesministerium für Bildung und Forschung/Deutsch-Israelische Projektkooperation, by the United States-Israel Binational Science Foundation, and by the Israeli Science Foundation. Special contributions from the Rosetrees Trust and the Ida Baruch fund are acknowledged. We thank Steve Wise and Tomer Fekete for helpful discussions and comments.

Correspondence should be addressed to Neta Zach at her present address: The Rockefeller University, 1230 York Avenue, New York, NY 10021. E-mail: nzach@mail.rockefeller.edu.

D0I:10.1523/JNEUROSCI.1965-08.2008

Copyright $\odot 2008$ Society for Neuroscience $\quad$ 0270-6474/08/289545-12\$15.00/0 cortices such as the primary visual cortex, there have been reports of specific changes in response properties corresponding to learning-specific perceptual discriminations (Schoups et al., 2001), but also reports of neuronal representations of novel features, especially if they are behaviorally relevant (Gilbert et al., 2001).

Several studies suggested that the motor and premotor cortices also undergo postlearning representational changes. For example, after adaptation to visuomotor rotations, neurons improve their coding of the direction used during the adaptation (Paz et al., 2003; Paz and Vaadia, 2004). In another study, after force-field adaptation, neurons change their preferred direction (Gandolfo et al., 2000; Li et al., 2001). Furthermore, studies using MRI and transcranial magnetic stimulation (TMS) methodologies have reported that the motor cortices are involved in consolidation of motor tasks after learning (Shadmehr and Holcomb, 1997; Muellbacher et al., 2002; Bütefisch et al., 2004; Robertson et al., 2004). However, the extent of these postlearning changes and their effect on baseline motor cortical representation remains unclear.

In this study, we explored the neuronal changes taking place during associative learning in which target color instructs movement direction. We found that a large fraction of M1 and premotor neurons exhibited responses specific to the learning task ("learning-related responses"). Moreover, these responses were maintained after learning, leading to manifestation of novel neuronal representations of arbitrary features such as the color of the target, in parallel to the ongoing representation of movement direction. 


\section{Materials and Methods}

Two female monkeys (Macaca fascicularis, $\sim 3$ $\mathrm{kg}$ ) were trained on an eight-direction centerout reaching task. They held a two-joint manipulandum at their elbow level with their left (dominant) hand, while their right hand was restrained. A horizontal plate concealed the arm and manipulandum from the monkey's sight. Animal care was in accordance with $\mathrm{Na}$ tional Institutes of Health guidelines and was approved and supervised by the Hebrew University committee on animal experiments.

\section{Recording}

Recording sessions started after training on the center-out task. Signals from 32 moveable electrodes placed in the motor cortices (see Fig. 4) on the monkey's right hemisphere were sorted and sampled at $25 \mathrm{kHz}$ (Alpha Omega).

\section{Trial flow}

As shown in Figure 1, each trial began with the screen display of an "origin" (a white circle) (Fig. 1a, left, depicted as gray). The monkey had to use the manipulandum to position a cursor (a white dot) (Fig. 1a, depicted as black) within this circle $(0.7 \mathrm{~cm}$ radius), for $750-1500 \mathrm{~ms}$ (varied randomly). Then, at target onset (TO), eight evenly spaced circles $(0.7 \mathrm{~cm}$ radius $)$ appeared. All circles were white (Fig. $1 a$, depicted in gray), with the exception of one colored circle (for example, Fig. 1a, the circle depicted in red). During the center-out task, the colored circle (red, green, blue, or magenta, selected pseudorandomly) served as the target. After a second delay of 750-1500 ms, the origin disappeared, cueing the monkeys to move ("GO signal"). The monkeys were rewarded with fruit puree if they moved in a straight trajectory (limited by an invisible corridor $1.4 \mathrm{~cm}$ in width), reached the target within $1 \mathrm{~s}$, and held the manipulandum there for an additional second. Performance was assessed using success rate (for bins of 10 trials) and several movement parameters, as follows: (1) reaction time, time from GO signal to movement onset (MO); (2) directional error, the angular distance between hand trajectory at peak velocity and the expected hand trajectory (averaged over centerout trials performed before learning); and (3) width and amplitude of velocity profiles. These parameters were compared before, during, and after learning.

\section{Session flow}

During recording, each session included three blocks. There was no cue to indicate block switching. The first and the third blocks consisted of the center-out task (eight targets, four colors, a total of 224 trials for each block). In the second (middle) block, we presented our main manipulation. We used two types of manipulations: the first was termed the "repetition block" and the second, the "learning block."

\section{Repetition blocks}

Before the monkeys had ever been exposed to the arbitrary association task (see Learning blocks, below), they were presented with control sessions in which they performed two blocks of the center-out task, before and after a repetition block. During the repetition block, the monkeys performed trials similar to those in the center-out task, except that only monkeys $M(\boldsymbol{f})$ and $K(\boldsymbol{g})$.

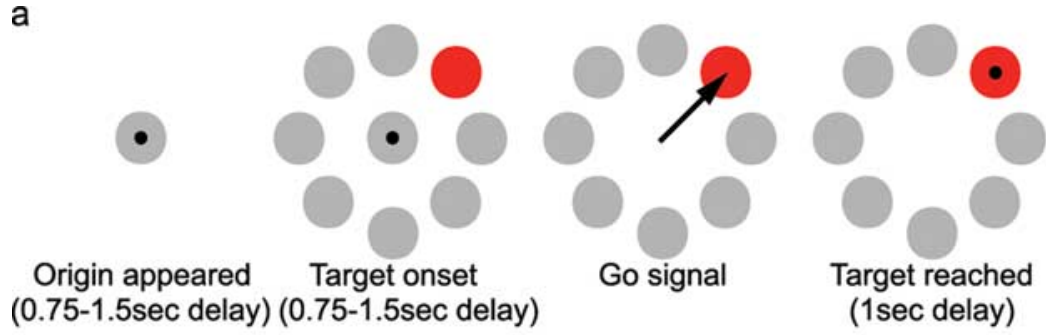

b
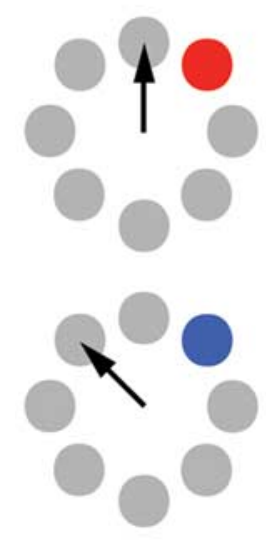

f

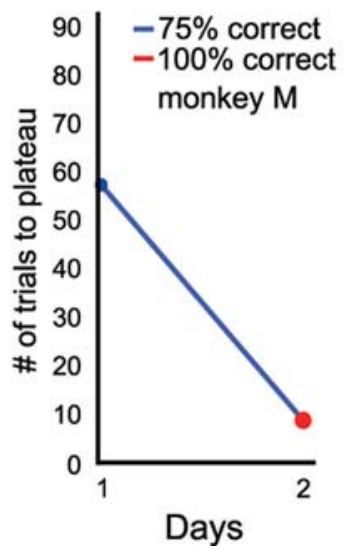

c
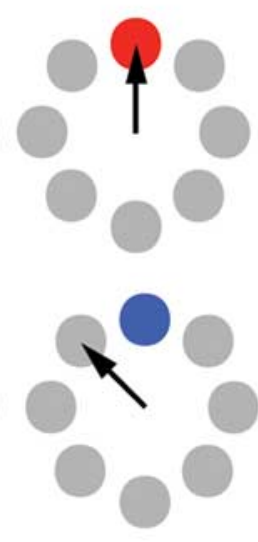

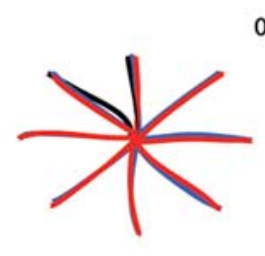

e

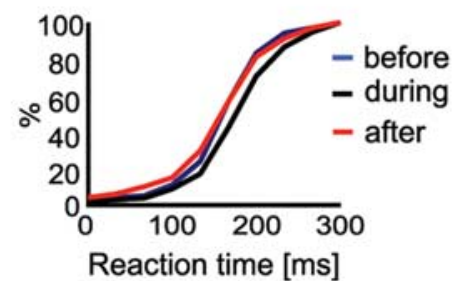

d

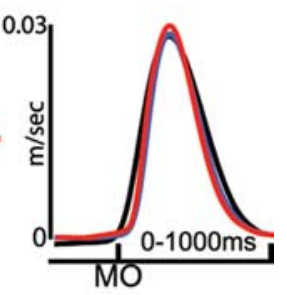

Figure 1. Experimental design and behavioral results. $\boldsymbol{a}$, Trial flow during the center-out task. Left to right: Each trial started by holding a cursor in a central origin (black dot and gray circle). After a $750-1500$ ms delay, 8 circles appeared, 1 colored (T0). After a second $750-1500$ ms delay, the origin disappeared, instructing the monkey to initiate movement (Go signal). The monkeys had to use the manipulandum to place the cursor in the target within $1000 \mathrm{~ms}$, and hold it there for $1000 \mathrm{~ms}$. $\boldsymbol{b}$, Arbitrary association task. The colored circle appeared pseudorandomly in one of two locations and in one of two colors. In contrast to the center-out task, the monkeys were not required to move into the colored circle. Rather, movement direction was instructed by the circle color (red, $90^{\circ}$; blue, $135^{\circ}$ ). c-e, Performance before (blue), during (black), and after (red) learning, showing examples of average movement trajectories (c) and velocity profiles ( $\boldsymbol{d}$ ) from one learning session (100 trials before, during, and after learning) and cumulative distributions of reaction times $(\boldsymbol{e})$ for all sessions. $\boldsymbol{f}, \boldsymbol{g}$, Retention of arbitrary association across learning sessions, as measured by the number of trials required to reach plateau performance of 75 and $100 \%$ success rates (stable for 20 trials) for

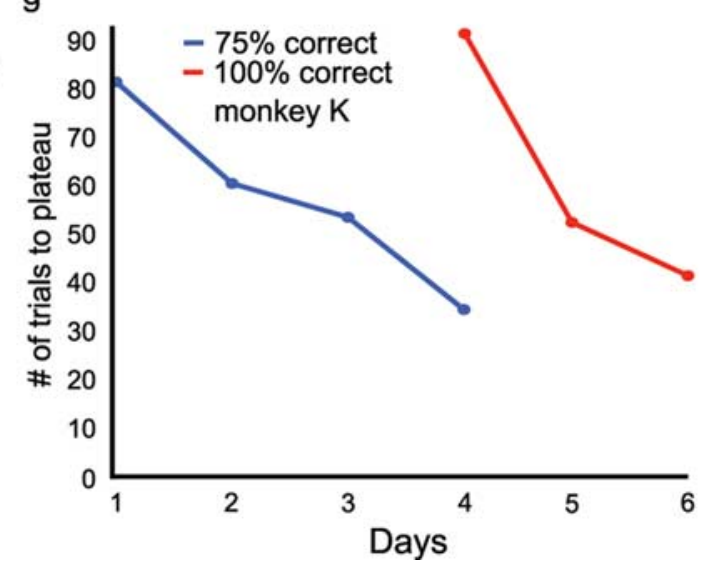

two of the eight possible movement directions and two of the four possible colors for the colored circle (target) were presented. These movement directions and target colors matched those that would later be used in the learning blocks. Repetition blocks included a total of 200 trials (randomized for circle colors and locations).

\section{Learning blocks}

After several days of recordings of repetition sessions, the monkeys began to perform the novel arbitrary association task. As in the repetition blocks described above, they performed two blocks of the center-out task, before 
and after a learning block. During the learning block, the monkeys learned an "arbitrary association task" (Fig. 1b), in which the color of the circle, not its location, specified the desired movement direction. A red circle instructed a movement (up) to a target at $90^{\circ}$ (Fig. $1 \mathrm{~b}$ ), whether it appeared at $45^{\circ}$ or $90^{\circ}$. The blue circle instructed a movement to a target at $135^{\circ}$ (up and to the left), also regardless of its location. In each learning block, the circles appeared in only two locations $\left(45^{\circ}\right.$ or $\left.90^{\circ}\right)$ as illustrated in Figure $1 b$. The monkeys had to learn to regard color as the relevant parameter and to find the two specific color-direction associations by trial and error. The learning block comprised 200 trials (randomized for circle colors and locations).

The monkeys were presented with this task day after day, with the same colors and locations, until there was no day to day improvement in performance. Improvement in performance was assessed by the number of trials required to reach stable (plateau) performance (for at least 20 consecutive trials). Therefore, all data in this paper relate to those learning sessions in which performance improved on the following day. Monkey $\mathrm{K}$ required six recording sessions to reach this level of performance, whereas monkey $\mathrm{M}$ required only $2 \mathrm{~d}$. Distributions of movement parameters (directional error, width, and amplitude of velocity profile and reaction times) were compared separately either for all trials (correct and errors) or for correct trials alone. Comparison was made using ANOVA (the Bonferroni correction for multiple comparisons was used for all calculations), and the distributions were compared using the KolmogorovSmirnov test.

\section{Data analysis}

Analyses of neuronal data were performed on the first 100 correct trials of each block of trials, using bins of $200 \mathrm{~ms}$ with a $50 \mathrm{~ms}$ interval, i.e., analysis of firing rates in the epoch of $0-200$, then $50-250$, etc., for the epochs from 0 to $750 \mathrm{~ms}$ after TO, and from $250 \mathrm{~ms}$ before to $500 \mathrm{~ms}$ after MO. To quantify the sensitivity of neurons to target colors and movement directions, we looked at the firing rate for each neuron and time bin in each block separately, and compared conditions using ANOVA, mutual information, and preferred direction (PD) analyses. All analyses were performed using the same neurons and the same number of trials before, during, and after learning.

ANOVA. We used multi-way ANOVA for target color, movement direction, and interactions, before and after learning. We also used ANOVA and a "discrimination ratio" to compare responses of neurons to the different blocks of trials (center-out as opposed to learning). The Bonferroni correction for multiple comparisons was used for all calculations. The discrimination ratio measures representations at the population level. It was defined as the number of discriminating neurons during each time bin (as calculated by ANOVA; significance threshold $p<0.01$ ) divided by the number of discriminating neurons expected by chance (using a bootstrap method with 500 repetitions of random shuffling). Significance was determined using Student's $t$ test $(p<0.01)$ between the observed discrimination and the discrimination expected by chance (the distribution of chance discriminations was produced using the bootstrap method). The discrimination ratio for target colors was termed "color sensitivity," the discrimination ratio for movement directions was termed "direction discrimination," and the discrimination ratio for interaction between movement directions and target colors was termed the "interaction ratio". The discrimination ratio for the different tasks was termed the learning-related response.

Mutual information. Mutual information was used to further assess discrimination between tasks, and was calculated using the standard formula (Papoulis 1984):

$$
I(x, y)=\sum_{x} \sum_{y} P(x, y) \log \left(\frac{P(x, y)}{P(x) P(y)}\right),
$$

where $x$ is the firing rate and $y$ is either the type of task, the type of association, or the target color. $P(x y)$ was calculated by assuming a Poisson distribution (the Poisson model well approximated the firing distribution for most cells), the mean of which was calculated across all trials (100 correct trials per block). The significance of mutual information for each neuron and each time bin was estimated using a bootstrap method (as in the ANOVA analysis). The significance of the change in mutual information of the entire neuronal population was compared before and after learning using ANOVA and the Kolmogorov-Smirnov test $(p<$ $0.01)$.

Correlations between behavioral performance and the neuronal responses were evaluated in the following way. The discrimination ratios for learning-related responses (discrimination between tasks) or for color sensitivity (discrimination of target color after learning) were assessed separately for each time bin in each learning session. These measures were then correlated, either to (1) the level of acquisition of the arbitrary association task (the number of trials required to reach stable performance for 20 trials) (Fig. $1 f, g$ ) or to (2) the retention of the arbitrary association task (the day-to-day changes in the number of trials required for stable performance) (the slope of Fig. $1 f, g$ ).

PD was calculated by fitting a cosine to the tuning curve of the responses of each cell for the eight possible movement directions (Georgopoulos et al., 1988) during the first 100 correct trials of the center-out block (before any learning was presented). To ensure validity of the PDs, only cells with $R^{2}>0.55$ to a cosine were included in this analysis $\left(R^{2}\right.$ as described by Paz et al., 2003; Paz and Vaadia, 2004). The uniformity of the PD distribution was evaluated by a Rayleigh test and homogeneity by Rao's spacing test $(p<0.01)$. The significance of the PD shift was determined by a bootstrap method with 500 repetitions of random shuffling (significance was determined using Student's $t$ test, $p<0.01$ ).

Correlations between firing rate changes during and after learning were performed the following way. (1) The magnitude of firing rate changes was calculated for each neuron during learning and after learning. Firing rate changes during and after learning were calculated only for trials in which movement directions and target colors matched those presented during learning (red, $90^{\circ}$; blue, $\left.135^{\circ}\right)$. (2) Firing rate changes were normalized by the magnitude of firing rates of that same neuron before learning (during the same trial types, red, $90^{\circ}$; and blue, $135^{\circ}$ ). (3) Correlations were calculated between the normalized response of each neuron during learning and its normalized response after learning. Correlations were computed separately for the TO and MO epochs.

Tuning curves and firing rate changes were calculated for a single time bin during each epoch: $350-550 \mathrm{~ms}$ after TO and -50 to $+150 \mathrm{~ms}$ relative to MO, because these were the time bins in which most cells were active. Similar results were obtained for other time bins as well.

\section{Results}

To investigate neuronal changes during associative learning, monkeys were presented with sessions comprising three blocks of trials: a block of a well known eight-direction center-out task, followed by a block of a novel arbitrary association task (learning block), and then another block of center-out task (see Materials and Methods). The associative task required moving in accordance with the target color (see Fig. $1 b$ and Materials and Methods). We examined changes in neuronal activity before, during, and after learning the novel task. All analyses were made on the same neurons, recorded throughout the entire session.

\section{Behavioral results}

Movement parameters

Figure 1, $c-e$, shows behavioral performance before, during, and after learning of the arbitrary association task. The analysis includes all trials in which the monkeys made movements, either correct or erroneous. We found that the learning process did not involve any changes in the movement parameters tested. The directional errors were similar before, during, and after learning in number and extent (Fig. $1 c$ shows average trajectories for one exemplary session, session III of monkey K. Full trajectories during three other exemplary sessions are shown in supplemental Fig. 1, available at www.jneurosci.org as supplemental material). The width and amplitude of velocity profiles were similar in the three blocks of trials (Fig. $1 d$ shows the average velocity profile for 
one exemplary session, session III of monkey K). Reaction times (RTs) (measured from onset of the GO signal) for monkey K were $200 \pm 12 \mathrm{~ms}$ (SEM), $212 \pm 14 \mathrm{~ms}$, and $203 \pm 10 \mathrm{~ms}$ before, during, and after learning, respectively. For monkey M, RTs were $195 \pm 8 \mathrm{~ms}, 209 \pm 15 \mathrm{~ms}$, and $200 \pm 11 \mathrm{~ms}$, respectively. Figure le shows the cumulative histograms of RTs (combined for all learning sessions).

ANOVA and the Kolmogorov-Smirnov test were used to compare these three parameters before, during, and after learning and showed that movement parameters did not differ $(p>$ 0.3 for all comparisons: all movement parameters, all blocks of trials, and all trials). This was also true when only the first 100 correct trials were compared [the trials for which neuronal activity was analyzed (see below); $p>0.3$ for all comparisons]. Also, we compared movement parameters during learning to movement parameters during the control repetition task (see Materials and Methods; comparison was made for all trials), but there were no distinguishable differences $(p>0.25)$.

\section{Acquisition of the arbitrary associations}

Although there were no changes in any of the movement parameters, performance on the arbitrary association task improved within and across sessions. The monkeys began each day by making many errors and progressed during each learning session to reach a plateau in the level of performance faster and at a higher level of performance. Supplemental Figure 1, available at www. jneurosci.org as supplemental material, shows learning curves for sessions I, IV, and VI of monkey K (learning blocks are depicted in the middle section). They show improvement in success rate, as well as rapid reduction of "perseveration errors" (errors caused by responses that followed the rules of the previous center-out task). Specifically, because one of the four possible trial types during associative learning (red target at $90^{\circ}$ indicating movement to $90^{\circ}$ ) was also identical to one of the center-out trials, we compared movement parameters during these trials to the other three types of learning trials. For a brief period of time at the beginning of the first learning session, the success rate was higher for this trial type, because of perseveration errors. On later trials and sessions, the success rate and movement parameters were similar in all four trial types presented during learning ( $t$ test, $p>$ $0.3)$.

Day-to-day changes were quantified by calculating the number of trials required to reach plateau performance on each session. Figure 1, $f$ and $g$, presents the day-to-day changes in this measure (two sessions for monkey $\mathrm{M}$ and six sessions for monkey $\mathrm{K})$. These improvements indicate that during the recording sessions, the task was in the process of being learned and consolidated in long-term memory (Brashers-Krug et al., 1996; Shadmehr and Brashers-Krug, 1997). The monkeys may have learned the task locally, for these particular colors, spatial locations, and associations. Alternatively, they could have learned the general rule "color instructs movement". We did not attempt to explore these possibilities.

\section{Performance of center-out task after learning}

Because an important goal of the study was to compare neuronal activity during performance of the center-out task before and after learning, special care was taken to compare behavior during these two blocks. For both monkeys, during the first two trials of the first postlearning block of the first learning session, there were occurrences of perseveration errors (as mentioned above, resulting from responding in accordance with the learned task that had just terminated). This is similar to the results of a human psycho- physics study of associative learning (Zach et al., 2005). These trials were excluded from analysis of neuronal activity. Such perseveration errors were not observed in the rest of the sessions (see supplemental Fig. 1, available at www.jneurosci.org as supplemental material).

Beyond perseveration, learning may also affect movement parameters, as in the case of visuomotor rotation and force-field adaptation, which manipulate the movement itself. After learning such tasks, there are short-lasting alterations (aftereffects) that may be reflected in curved trajectories of the arm. To examine the possible occurrences of aftereffects, we looked at directional errors for the directions used during learning, compared with directions not used during learning. Supplemental Figure 2, available at www.jneurosci.org as supplemental material, shows directional errors for the first postlearning trial for each target color and movement direction for three exemplary sessions. It shows that besides a perseveration error on the first session, the degree of directional errors in movement directions or target colors that were used during learning was not significantly greater than the extent of directional errors of movements in other directions and colors. This is also in line with human psychophysics results (Zach et al., 2005), suggesting that learning a color-direction association task does not affect movement parameters. This can be explained by the nature of the arbitrary association task, which does not involve manipulation of movement parameters such as direction.

In sum, although the monkeys learned the association task, movement parameters did not show statistically significant changes across blocks, recording sessions, movement directions, or target colors. These findings indicate that movements during learning were indistinguishable from ones made during the center-out task. Also, postlearning performance of the center-out task was similar to prelearning performance.

\section{Neuronal activity}

The data included 256 cells, with 140 cells (104 from monkey K, 36 from monkey $\mathrm{M}$; eight different sessions) recorded during learning sessions and 116 cells recorded during repetition sessions ( $n=88$ for monkey M, $n=28$ for monkey $\mathrm{K}$ ). Neuronal activity was recorded in the upper surface of M1 and in two premotor areas [premotor dorsal (PMd) and premotor ventral $(\mathrm{PMv})$ ] (Fig. 4, see surface map for monkey K). The activity of these neurons in the first 100 correct trials during each block was examined using $200 \mathrm{~ms}$ time bins in two main epochs of each trial: from 0 to $750 \mathrm{~ms}$ after target onset (this epoch is referred to as TO) and from $250 \mathrm{~ms}$ before movement onset to $500 \mathrm{~ms}$ after movement onset (this epoch is referred to as MO) (see Materials and Methods).

All neurons included in this analysis had (1) a stable firing rate throughout the entire session, measured by comparing responses during the hold period (750 ms before TO) throughout the three blocks of each session; (2) a firing rate of $>1 \mathrm{~Hz}$ during that hold period; and (3) a significant response (firing rate changes) during both the TO epoch and the MO epoch, compared with the hold period. Because there were no differences in firing rate, variability, or any neural parameter tested, the neurons of the two monkeys were pooled.

\section{Learning-related responses}

To illustrate the neuronal activity discriminating the center-out task and the learning task, Figure 2 shows exemplary peri-event time histograms for two neurons, one during TO (top; recorded from PMv) and the second during MO (bottom; recorded from 


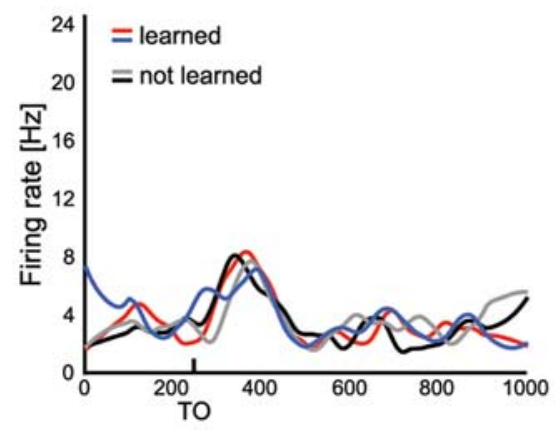

b

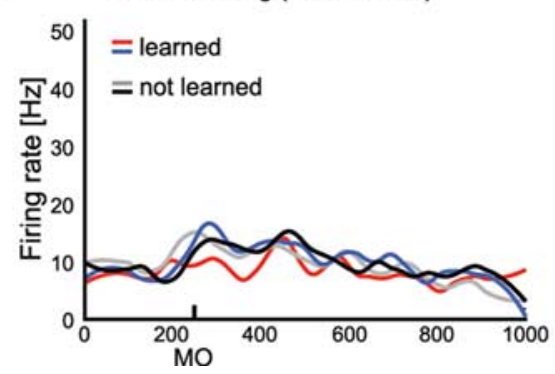

During learning

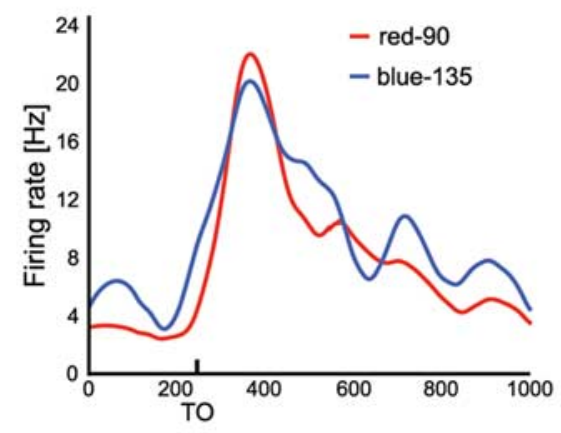

During learning

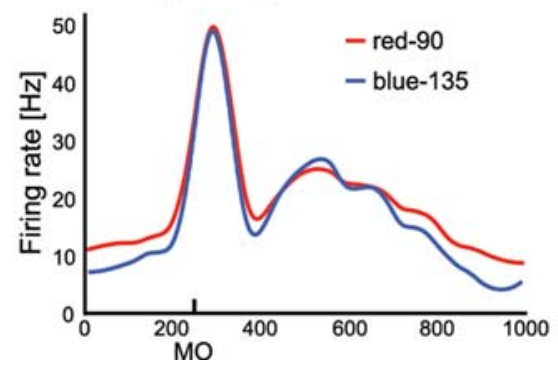

After learning (all directions)

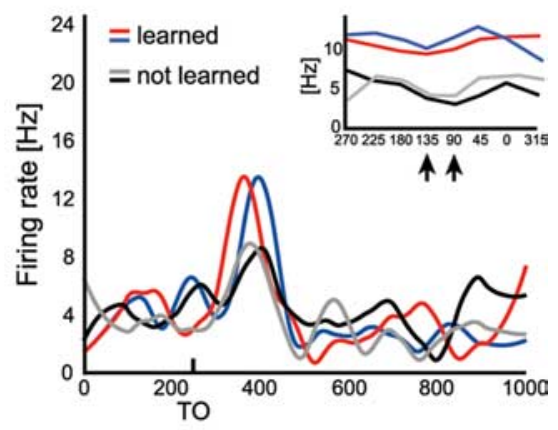

After learning (all directions)

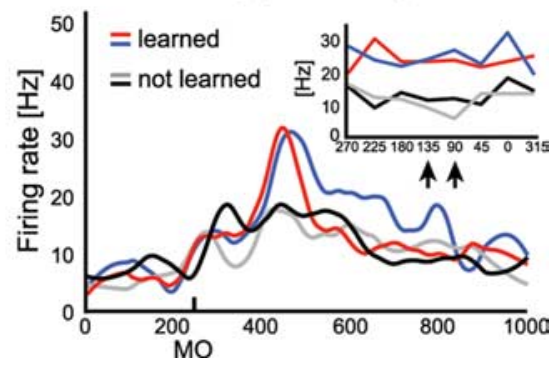

Figure 2. $\quad \boldsymbol{a}, \boldsymbol{b}$, Examples of two neurons showing learning-related responses and color sensitivity. The responses of one cell are shown around TO ( $\boldsymbol{a})$ and the other cell, around $\mathrm{MO}(\boldsymbol{b})$. Responses were smoothed using bins of $50 \mathrm{~ms}$. Left, Responses to different target colors before learning (red and blue, the colors that would be presented in the learning task; black and gray, representing magenta and green, respectively, colors presented in the center-out but not the learning task). Responses to the target colors red and magenta (depicted as black) are shown only for trials in which movement was made to $90^{\circ}$, and responses to the target colors blue and green (depicted as gray) are shown only for trials in which movement was made to $135^{\circ}$. Middle, Responses during learning of color-direction associations (red, $90^{\circ}$; and blue, $135^{\circ}$ ). Note the differences in these responses from the responses to the same movement directions and target colors during the center-out task. Right, Responses to target colors after learning of the color-direction association task. Note the maintenance of the elevated response for the target colors that were relevant during learning (this response was termed color sensitivity). Inset, Tuning curves for the different target colors after learning. Arrows mark the movement directions used during the arbitrary association task. Note that color sensitivity was similar across all movement directions. The cell in $\boldsymbol{a}$ was recorded from PMv and the cell in $\boldsymbol{b}$ from M1.

M1) before, during, and after learning. Raster plots of these neurons are shown in supplemental Figure 3, available at www. jneurosci.org as supplemental material. The histograms show responses to the different target colors before, during, and after learning. Responses during the center-out task are shown specifically for the directions that were used during learning (red and magenta at $90^{\circ}$, green and blue at $135^{\circ}$ ). In other words, in all parts of the figure, red lines depict trials in which target color was red and movement was made to $90^{\circ}$, and blue lines depict trials in which target color was blue and movement was made to $135^{\circ}$. However, responses during the center-out task before learning were largely different from responses during learning; although movement directions and target colors were similar, the responses distinguished between the center-out task and the learning task. These discriminative responses were termed learningrelated responses.

We assessed learning-related responses of all of the cells recorded during the learning sessions ( $n=140$ cells), by comparing responses during the arbitrary association and center-out tasks. Responses were compared for each neuron, for trials with the same target colors and movement directions (i.e., red, $90^{\circ}$ and blue, $135^{\circ}$ ), either on the center-out task or on the novel arbitrary association task, using ANOVA. A neuron having responses that discriminated between the two tasks was classified as a neuron showing learning-related responses. Note that all neurons that responded differently to the two tasks did so while maintaining the same background firing rate throughout the session (neurons that failed to meet these criteria were considered instable and were excluded from further analysis; see above). Also, note that responses were made to the same target colors and movement directions. After computing the discrimination for each cell, we calculated a discrimination ratio for the entire neuronal population (the ratio of the number of cells classified as learning related by the ANOVA to the number expected by chance; see Materials and Methods). The discrimination ratio was calculated separately for each $200 \mathrm{~ms}$ time bin.

As shown in Figure $3 a$, the population showed a significant increase in discrimination ratios $(p<0.01)$, across all time bins, reaching up to 18 -fold more than chance level during both TO and MO. The numbers indicate that up to $49 \%$ of the neurons showed significant learning-related responses (51 of 104 for monkey K, 17 of 36 for monkey M).

We validated the results by several post hoc analyses. First, we compared the level of learning-related responses on the different learning sessions and found them to be similar $(p>0.5)$. Also, we assessed whether there were any observable changes in the amount of learning-related responses throughout the learning block, by calculating discrimination ratios separately for the two halves of the learning block (comparing the first 50 trials of the learning block to the last 50 trials of the learning block). No such changes were observed $(p>0.5)$.

A more specific test of the learning-related responses was performed by looking at learning trials in which the target location, its color, and the required response were identical to that of center-out trials (red at $90^{\circ}$ instructed movement to $90^{\circ}$, one of the four possible trial types during learning, as shown in Fig. 1b). We compared the responses during those trials to responses during other learning trials or during the matching center-out trials. We found that of 68 learning-related neurons, 66 (97\%) responded during this trial type in accordance with their learning- 
a

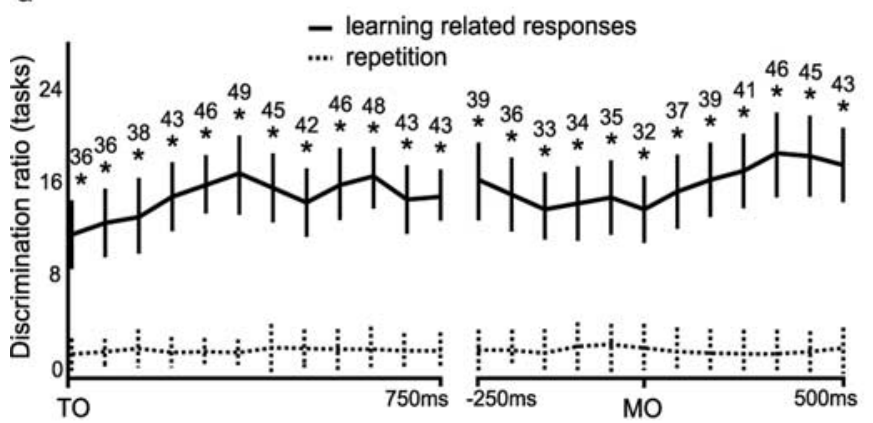

b

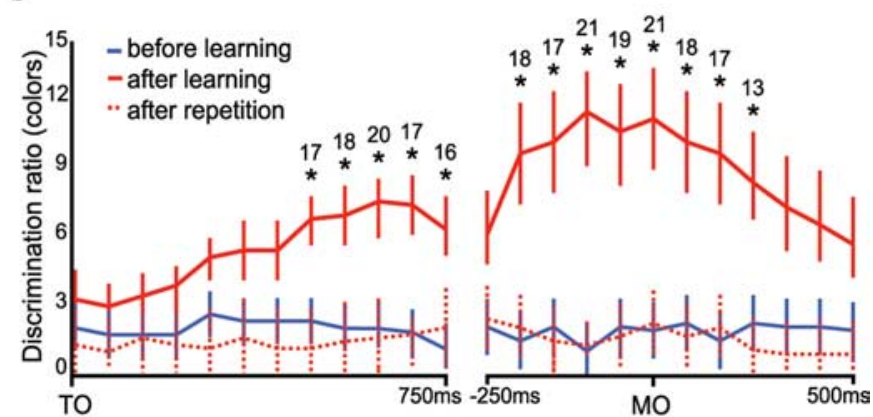

C

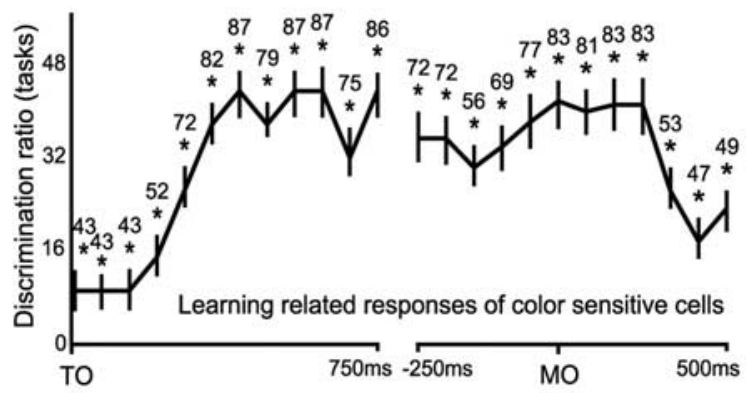

d

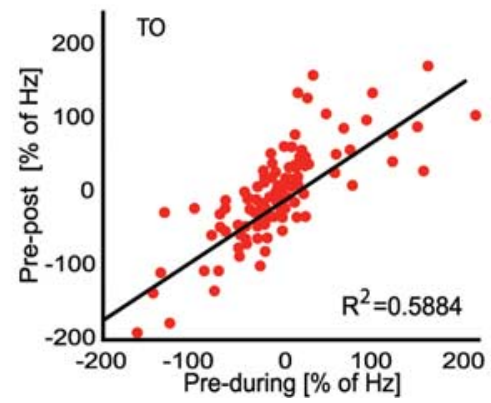

e

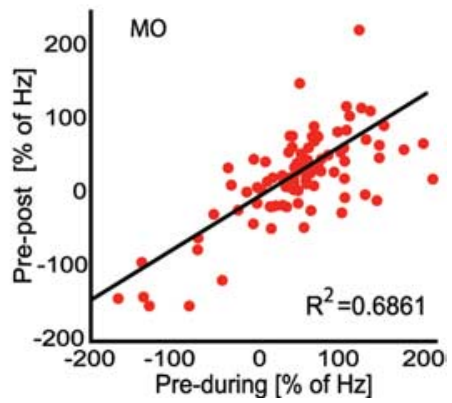

Figure 3. Learning-related responses and color sensitivity of the neuronal population. $\boldsymbol{a}, \boldsymbol{b}$, Learning-related responses ( $\boldsymbol{a}$ ) (solid lines) were quantified by discrimination ratios (discrimination between tasks for the entire neuronal population, relative to chance levels, $n=140$ cells) (see Materials and Methods), calculated for $200 \mathrm{~ms}$ time bins, relative to T0 (left) and M0 (right). Error bars are \pm SEM. Stars indicate significance $(p<0.01)$. The numbers mark the percentage of neurons responding discriminatively to the same movement directions and target colors when presented in the learning task as opposed to center-out task. Dashed lines depict discrimination ratios calculated during the control repetition sessions ( $n=116$ cells) (see Materials and Methods). $\boldsymbol{b}$, Color sensitivity after learning. Color sensitivity was quantified by discrimination ratios (discrimination between target colors for the entire neuronal population, relative to chance levels, $n=140$ cells) (see Materials and Methods) before learning (blue), after learning (red), and after the control repetition session (gray). The numbers mark the percentage of neurons responding discriminatively to the different target colors after learning. Notation is the same as in $\boldsymbol{a} . \boldsymbol{c}-\boldsymbol{e}$, Evidence showing that color sensitivity is a continuation of the learning-related responses after learning has ended. $\boldsymbol{c}$, Discrimination ratios for learning-related responses (as in $\boldsymbol{a}$ ) calculated for the specific subpopulation of neurons that showed color sensitivity after learning (depicted in $\boldsymbol{b}$ ). Notation is as in $\boldsymbol{a}$. Note that almost all color-sensitive neurons also showed learning-related responses. $\boldsymbol{d}, \boldsymbol{e}$, Correlations between each neuron's learning-related responses and color sensitivity, during the TO (d) and $\mathrm{MO}$ (e) epochs. $x$-Axis, Learning-related responses (firing rate changes for the same movement directions and target colors during learning, normalized by firing rate to these trial types before learning). $y$-Axis, Color sensitivity (firing rate changes to these target colors and movement directions after learning, normalized by firing rate to these trial types before learning). Note that the two phenomena were highly correlated.

related responses and not their center-out responses. This result strongly supports the notion that learning-related responses indeed reflect the context of the task rather than specific task parameters.

To further validate these results, we compared them to results obtained in sessions of a control task, called "repetition sessions" ( $n=116$ cells, four sessions; see Materials and Methods). Repetition sessions were conducted before the monkeys had any exposure to the arbitrary association task. All procedures and analyses were as described above, except that instead of a block of the arbitrary association task, the monkeys performed a repetition task: a center-out task with the same two target colors and two movement directions that were later used during learning of the arbitrary association task. We compared neuronal activity for the same movement directions and target colors during the centerout task versus the repetition task. We found that the number of neurons discriminating the repetition task from the center-out task never rose above a chance level (Fig. 3a, black dashed lines) $(t$ test, $p<0.01$ from arbitrary association sessions). These results show that learning-related responses cannot be ascribed to the mere presentation of only two movement directions rather than eight directions in the center-out task.

To further validate these results, we also computed the mutual information (Papoulis, 1984; Hatsopoulos et al., 1998) between neural activity and the task performed. Whereas ANOVA is used to determine discriminative responses in general, the mutual in- formation approach provides more precise information about the reliability of the discriminative responses. Indeed, the mutual information regarding the type of task being performed was high (see supplemental Fig. $4 a$, available at www.jneurosci.org as supplemental material), showing that the learning-related responses were consistent and reliable.

To conclude, although movements during center-out and arbitrary association tasks were made to the same directions with the same target colors, many neurons in the motor and premotor cortices responded to them differentially during the different tasks, showing learning-related responses.

\section{Sensitivity to target color after learning}

As shown in Figure 2 (right column), after learning, on returning to the well known center-out task, some of the neurons with the learning-related responses maintained a trace of these responses by evolving a novel representation of target colors. These neurons responded differently to the target colors that were used during learning (red and blue) as opposed to the colors not used during learning (the colors that were only used during the center-out task). We termed this response color sensitivity, because the neurons responded to the instructive properties of the target color. Directional tuning curves, shown in the insets of Figure 2 (right), indicate that color sensitivity was evident across all movement directions.

To evaluate the nature of this color sensitivity, we first per- 
formed an ANOVA, comparing the responses of the neurons to the four possible target colors before and after learning. As shown in Figure $3 b$, after learning the color-direction association, the neurons discriminated colors: before learning, the discrimination ratio never rose above chance levels (blue lines). However, after learning (red lines), there was a significant increase in the discrimination ratios. The discrimination of target color reached as high as sixfold more than expected by chance during TO (up to $20 \%$ of the neurons; 24 of 104 for monkey K, 4 of 36 for monkey $\mathrm{M}$ ), and up to a tenfold increase during $\mathrm{MO}$ (up to $21 \%$ of neurons; 23 of 104 for monkey $\mathrm{K}, 7$ of 36 for monkey $\mathrm{M}$ ).

As a control, we also compared discrimination of target color before and after performing the repetition task. We found that the after-repetition discrimination ratios did not rise above a chance level (Fig. 3b, dashed red lines), showing that neurons became sensitive to the target colors only if these features were used during the association learning. To further verify these results, we computed mutual information between neuronal activity and target color. The results of this analysis are shown in supplemental Figure 4, available at www.jneurosci.org as supplemental material, demonstrating that neuronal activity after learning, but not before, was informative regarding target colors.

As shown in the examples in Figure 2, color sensitivity stemmed from responses discriminating the colors used during the task from the colors not used during learning (used only during the center-out task). This result holds for the vast majority of color-sensitive cells; a post hoc $t$ test analysis determined that in all time bins, $65-100 \%$ of the color-sensitive neurons in fact discriminated the colors used during learning (red and blue) from the colors not used during learning (green and magenta). Also, note that as in the case of the learningrelated responses, there was no tendency to respond differently to red as opposed to blue, the two colors associated to different movements. Finally, we calculated the discrimination ratio separately for the first and second halves of the block of the center-out task after learning, for each of the different learning sessions, and found that color sensitivity was maintained at the same level throughout the postlearning block $(p>0.5)$ (results not shown). These results support the notion that color sensitivity was maintenance of the learning-related responses.

To further test this possibility, we assessed the relationship between learning-related activity and postlearning color sensitivity. As shown in Figure 3c, most (75-90\%) of the color-sensitive neurons also showed learning-related responses. Furthermore, we assessed the correlation between the magnitude of learningrelated responses by each neuron (firing rates during learning, normalized by responses to same movements and colors before learning) to the magnitude of its color sensitivity (firing rates after learning, normalized by responses to these target colors and movement directions before learning). As shown in Figure 3, $d$ and $e$, the firing rate of each neuron during learning predicted its responses after learning well $\left(R^{2}=0.5884\right.$ and $R^{2}=0.6861$ during TO and MO, respectively). These results suggest again that color sensitivity was in fact maintenance of the learning-related responses.

We conclude that traces of learning-related responses were maintained after learning, during performance of the center-out task, in which the colors were task-irrelevant. This shows that motor and premotor neurons can maintain responses to features relevant to behavior, even sensory features such as colors that are not normally represented in the motor cortex.

\section{Cortical location of discriminative neurons}

Neurons with learning-related responses and color sensitivity were found in both M1 and premotor areas of the two monkeys. Figure $4 a$ shows, for monkey $\mathrm{K}$, the recording sites in which cells with learning-related responses were found (black dots; $n=55$ sites) and in which color-sensitive cells were found (red dots; $n=$ 23 sites). Gray dots mark sites in which no discrimination was found ( $n=41$ sites). Note that the total number of sites in the figure is smaller than the number of neurons ( $n=104$ for monkey K) because each electrode recorded up to two neurons simultaneously, and some locations were repeated in more than one session.

Figure $4 b$ shows sites in which intracortical microstimulations induced movements around the shoulder or elbow at $<20 \mu \mathrm{A}$ of current ( $n=36$ sites). These sites were considered to be within M1; the more rostral sites were classified as being within the premotor cortex. Among them, sites that were located medial to the genu of the arcuate sulcus were considered as PMd sites, and sites residing laterally to the genu were considered PMv sites. The borders between areas are marked by dashed black lines in Figure 4, $a$ and $b$. As shown in Figure $4 a$, learning-related responses and color sensitivity prevailed in all three regions. This was also true for monkey $\mathrm{M}$ : there were 22 sites with neurons that showed learning-related responses and 7 sites with color-sensitive neurons, all distributed across M1 and premotor areas (results not shown).

To further examine any differences between the areas, we recalculated the discrimination ratio for the learning-related responses and color sensitivity separately for neurons in each site (M1, $n=60$ cells; PMv, $n=63$ cells; PMd, $n=17$ cells from the two monkeys). The results are shown in Figure $4 c$ for learningrelated responses and Figure $4 d$ for color sensitivity, with dashed lines representing the discrimination ratios for a single cortical area, versus solid lines representing the discrimination ratios of the entire neuronal population (same as Fig. $3 a, b$ ). In all comparisons, there were no differences between the magnitude of discrimination ratios in one brain area compared with the entire neuronal population (ANOVA, $p>0.2$ ). Also, there were no differences when comparing the three areas to each other $(p>$ 0.2 ) This shows that learning-related responses and color sensitivity were widely distributed, extending from the central sulcus to the arcuate sulcus and including both the ventral and dorsal premotor areas.

\section{Representation of movement direction is unaffected}

We examined whether learning novel associations between target colors and specific movement directions had any effects on the representation of movement directions by $\mathrm{M} 1$ and premotor cortex. We measured direction representation using ANOVA and the discrimination ratio for the different movement directions, before and after learning, and for a possible interaction between movement direction and target color. As shown in Figure $5 a$, the discrimination ratios for the different movement directions were similar, before and after learning, for both the TO and MO epochs $(p>0.2)$. In addition, there were no interactions between color and direction (Fig. $5 a$, inset). This shows that representation of direction was not affected by the emergence of color sensitivity.

To further probe the direction representation of the cells, we calculated the cells' directional tuning and PD. Figure $5 b$ shows the PDs of cells with cosine fit of $>0.55$ (as described by Paz et al., 2003, Paz and Vaadia, 2004) (see Materials and Methods), before (blue lines) and after (red lines) learning. The direction of each 

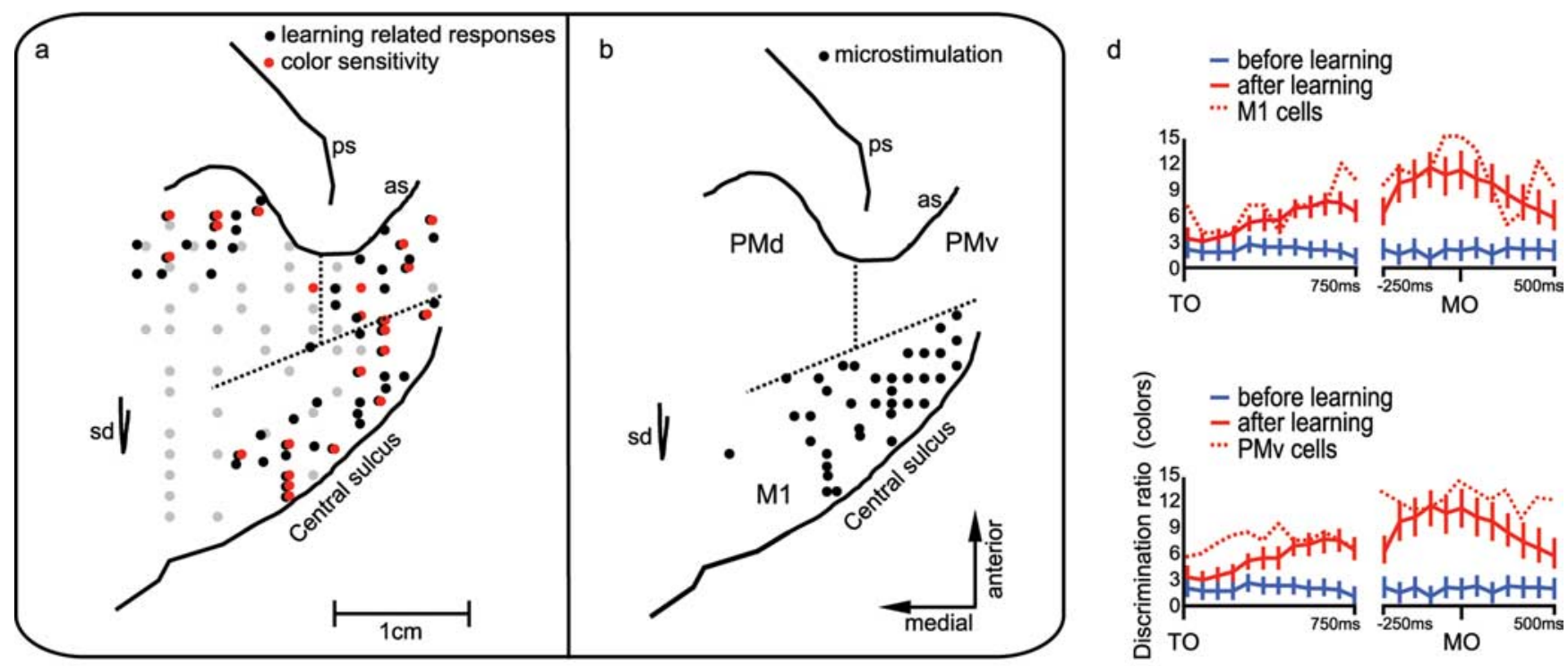

c

\section{- learning related responses}

-.. (specific cortical locations)
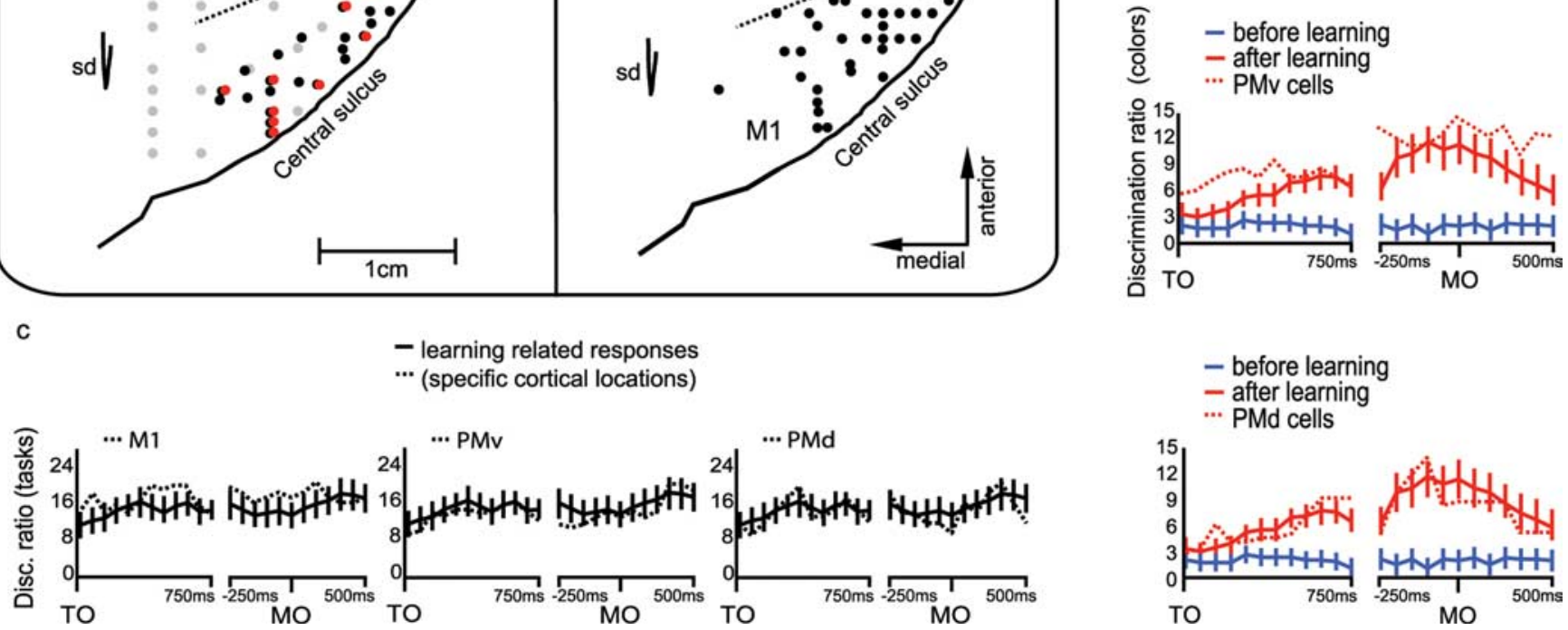

Figure 4. $\boldsymbol{a}, \boldsymbol{b}$, Locations of neurons showing learning-related responses or color sensitivity, across $\mathrm{M} 1$ and premotor cortex. Cortical locations of recording sites $(\boldsymbol{a})$ and microstimulation $(\boldsymbol{b})$, both from monkey K, are superimposed on a surface map, extracted from MRI analysis (BioSpec 4.7 T; Bruker) and verified by skull endocast analysis. as, Arcuate sulcus; ps, principal sulcus; sd, superior precentral dimple. Dashed lines classify areas as M1, PMd, or PMv. $\boldsymbol{a}$, Locations of neurons showing learning-related responses (black dots; $n=53$ sites), neurons showing color sensitivity after learning (red dots; $n=21$ ), and nondiscriminative neurons (gray dots; $n=41$ ). $\boldsymbol{b}$, Microstimulation map. Black dots, Locations in which intracortical microstimulation of $<20 \mu A$ induced movements around the shoulder or elbow $(n=36)$. $\boldsymbol{c}, \boldsymbol{d}$, Learning-related responses (c) and color sensitivity (d), recalculated separately for neurons recorded from M1 $(n=60$ cells), PMv $(n=63)$, and PMd $(n=17)$ of both monkeys. Responses are marked by dashed lines and compared with responses of the entire neuronal population (marked by solid lines; $n=140)$ (same as Fig. 3a,b). c, Learning-related responses for neurons recorded from the different areas. Notation is as in Figure $3 a$. $\boldsymbol{d}$, Color sensitivity for neurons recorded from the different areas. Notation is as in Figure $3 b$. Note that the learning-related responses and color sensitivity were distributed evenly across M1 and premotor cortex.

line represents the PD of one tuned cell, and its length represents $R^{2}$ to cosine. The distribution of PDs was uniform and homogeneous during both epochs (Raleigh $p=0.37$ and $p=0.41$, Rao $p=0.42$ and $p=0.35$ for $\mathrm{TO}$ and $\mathrm{MO}$, respectively). Also, there was no difference in the number of neurons with cosine-like directional tuning ( $n=47$ cells before vs $n=46$ cells after learning for the TO epoch, $n=45$ before and after learning for MO) and no significant $\mathrm{PD}$ shift $(n=3$ cells with significant $\mathrm{PD}$ change during TO, $n=5$ during MO). Thus, learning a color-associative task did not induce changes in the representation of direction in the motor areas studied.

\section{Learning-related response and its maintenance are independent of movement direction}

As shown above (Fig. $1 c-e$ ), trajectories were not affected by learning, and color sensitivity was apparent across all movement directions (see examples in Fig. 2 right, inset). This raises the possibility that learning-related responses and color sensitivity were direction independent. Therefore, we explored the connection of these neuronal responses to movement directions and to the cells' PD. Of the entire population of tuned cells depicted in Figure 5, $b$ and $c$, Figure $6 a$ shows the PD distributions of two subgroups of tuned cells: tuned cells that showed learning-related responses (black lines) and tuned cells that showed both learning-related responses and color sensitivity (red lines). PDs are shown separately for the TO epoch (left, $n=38$ and 15 cells, respectively) and MO epoch (right, $n=41$ and 21 cells, respectively). The PDs of the two groups were uniformly and homogeneously distributed (for learning-related responses, Raleigh test $p=0.68$ and $p=0.51$, Rao $p=0.56$ and $p=0.53$ for TO and MO, respectively; for color sensitivity, Rayleigh $p=0.23$ for TO, $p=$ 0.31 for the MO epoch, and Rao's spacing test $p=0.3$ and $p=$ 0.37 , respectively). Also, there were virtually no shifts in PD after learning in comparison with before learning (only two learningrelated cells showed a significant change of their PD during TO, and three learning-related cells during MO). This shows that learning-related responses and color sensitivity were not specific to cells with particular PDs.

We also explored whether color sensitivity was more prevalent for movement directions that were used during learning. To do so, we recalculated discrimination ratios for color sensitivity during the postlearning center-out task, omitting the trials in which movements were made to one of the two movement directions that were used during learning (discrimination ratios were calculated for six of eight directions and all four possible target colors). As shown in Figure $6 b$, color sensitivity after the exclusion (dashed lines) was indistinguishable from color sensitivity calculated for all trials (solid lines). This shows that color sensitivity was generalized well beyond the movement directions presented during learning. 
a

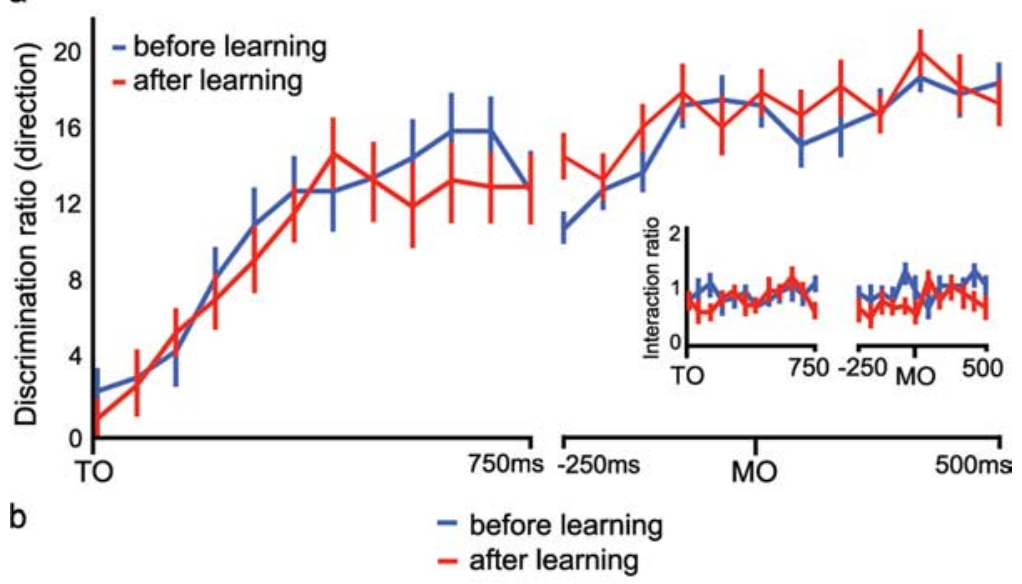

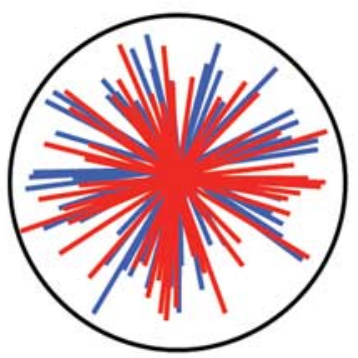

TO

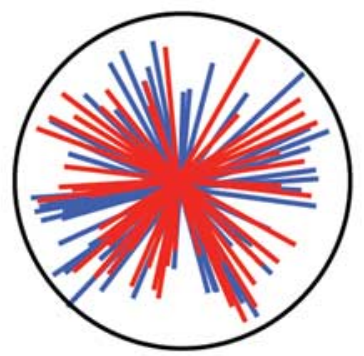

MO

Figure 5. Representation of movement direction is unaffected by learning. $\boldsymbol{a}$, Discrimination of movement directions was quantified by discrimination ratios (discrimination between different movement directions relative to chance level, $n=140$ cells) (see Materials and Methods) before and after learning. Inset, Interaction ratio (ratio of number of cells responding discriminatively to specific combination of movement directions and target colors, relative to chance levels; $n=140$ ) between movement directions, before and after learning. Notation is as in Figure $3 b . \boldsymbol{b}$, Distribution of PDs before (blue lines) and after (red lines) learning, during TO (left) and MO (right) epochs. The direction of each line represents a cell's PD, and its length is proportional to the $R^{2}$ for a fit to a cosine function (the radius of the black circle is the maximal possible value of $R^{2}=1$ ). Only cells with cosine fit of $R^{2}>0.55$ during the center-out trials before learning were considered tuned and included in this analysis ( $n=47$ and 45 cells for $\mathrm{TO}$ and $\mathrm{MO}$, respectively).

To further elucidate any connection to the cells' PD, we recalculated discrimination ratios for learning-related responses and color sensitivity for specific subpopulations of neurons (Fig. $6 c-e)$. Dashed lines show the recalculated discrimination ratio, compared with the solid lines representing the discrimination ratio of the entire population (same as Fig. 3a,b). Discrimination ratios were recalculated specifically for cells that were tuned in the prelearning block ( $n=47$ and 45 cells for TO and MO, respectively) versus specifically for nontuned cells ( $n=93$ and 95 cells respectively) (Fig. 6c) or specifically for tuned cells having a PD that was close to the movement direction used during learning ( $n=12$ and 11 cells, respectively) (Fig. $6 d$ ). In all of these subpopulations, discrimination ratios were indistinguishable from that of the entire neuronal population or from each other (ANOVA, $p>0.25$ ). Finally, we recalculated the discrimination ratios for tuned cells, only during trials in which movements were made to directions far from the cells' $\mathrm{PD}\left(>22^{\circ}\right)$ (Fig. 6e). Again, discrimination ratios were similar to those found for all trials. This shows that learning-related responses and color sensitivity were not specific to any of the subgroups of neurons tested here. Note that this is also in line with the examples in Figure 2 and supplemental Figure 3, available at www.jneurosci.org as supplemental material, showing two nontuned cells with learningrelated responses and color sensitivity.

Together, these results suggest that learning of the arbitrary association task, in which the movement direction itself is not manipulated, did not affect the cells' directional tuning, and the neural responses (learning-related responses and color sensitivity) were unrelated to the cells' PD.

\section{Discussion}

The results show that neurons in $\mathrm{M} 1$ and premotor cortex can rapidly become sensitive to various novel features such as color, if such features are relevant for encoding an upcoming movement. Moreover, these motor neurons maintained their novel representations after learning, even when they were no longer relevant in instructing movement. Specifically, we found that neurons in these areas showed learning-related responses; i.e., they responded differentially during the learning task as opposed to the center-out task executed before learning. On returning to the center-out task, many neurons maintained some of the learning-related responses by responding differently to the target colors that were relevant during learning (although the learning block was terminated and target colors were irrelevant to successful completion of the task).

\section{Learning-related responses}

There have been reports showing changes in single neuron firing rate during learning for both M1 (Gandolfo et al., 2000; Li et al., 2001; Paz et al., 2003) and nonprimary motor areas (Paz et al., 2003; PadoaSchioppa et al., 2004). These recordings were obtained during nongeneralized tasks such as visuomotor rotation, and indeed, the neuronal responses were specific, exclusive to a restricted subpopulation of neurons, for example, neurons having a PD that was close to the directions used in a rotation task (Paz et al., 2003). This is similar to results from primary sensory areas showing highly specific changes in their basic discriminative properties during learning of highly specific perceptual tasks (Gilbert et al., 2001; Schoups et al., 2001; Paz et al., 2004).

However, in the primary sensory areas, there are also reports of rapid large-scale and general representational changes (Crist et al., 2001; Gilbert et al., 2001; Blake et al., 2002; Li et al., 2004) during performance of more complex tasks such as responding to complex stimulus configurations. Such responses were contextual and task dependent, and did not affect the basic tuning of the relevant neurons. The learning-related responses reported here parallel these findings in the motor cortices; as expected from contextual responses, they were found in a large portion of M1 and premotor neurons, they appeared regardless of the cells' basic tuning (Fig. 6), and they did not hinder the basic direction representation of the motor cortical neurons (Fig. 5). These results demonstrate that the motor cortices have the ability to form novel representations of arbitrary features, if they are behaviorally relevant.

The apparent difference between our results and the specific neural changes found in M1 in the past can be explained by the 
a

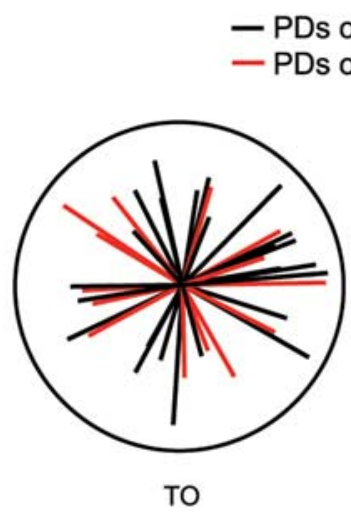

C

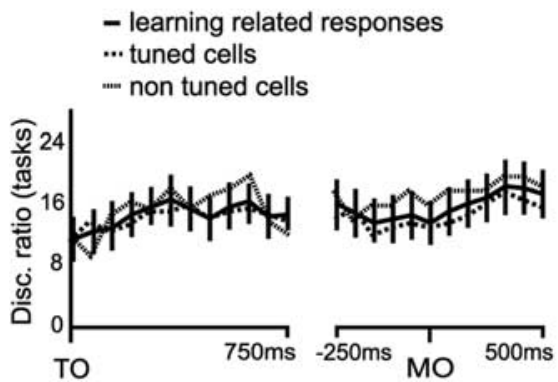

... tuned cells

-..' non tuned cells

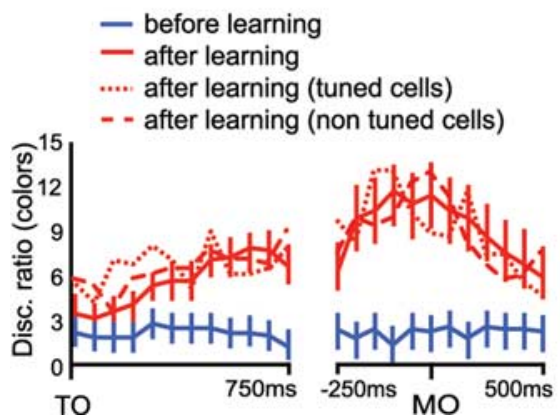

b

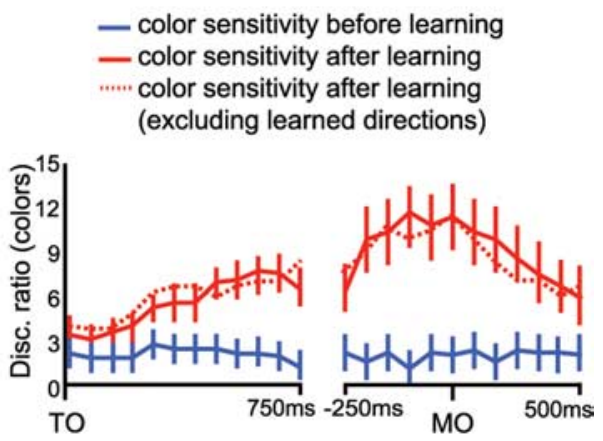

e

$$
\begin{aligned}
& \text { - learning related responses } \\
& \text {-. cells with relevant PD }
\end{aligned}
$$

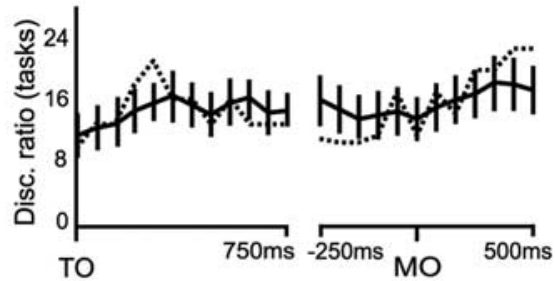

- learning related responses

-. (excluding PD)

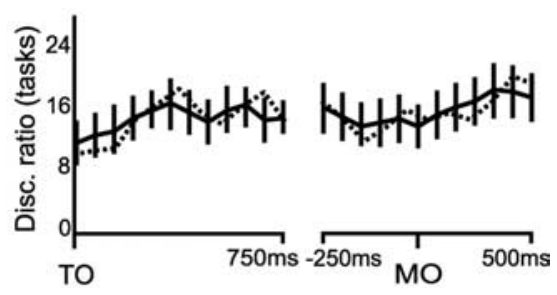

$$
\begin{aligned}
& \text { - before learning } \\
& \text { - after learning } \\
& \ldots . . \text { after learning (cells with relevant PD) }
\end{aligned}
$$

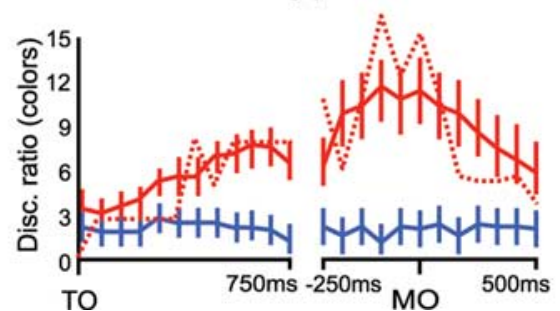

- before learning

- after learning

.... after learning (excluding PD)

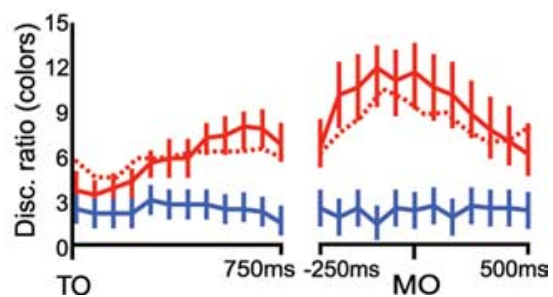

Figure 6. $\boldsymbol{a}-\boldsymbol{e}$, Learning-related responses and color sensitivity are independent of movement direction. $\boldsymbol{a}$, Distribution of PDs for neurons that were tuned before learning and also showed learning-related responses (black lines), during the TO (left; $n=38$ cells) and MO (right; $n=41$ ) epochs. Red lines indicate neurons that were also color sensitive after learning ( $n=15$ during T0, $n=21$ during M0). Notation is as in Figure $5 b$. Note that PDs of learning-related and color-sensitive neurons are distributed evenly (see Results for statistics). $\boldsymbol{b}$, Color sensitivity calculated specifically for the movement directions that were not used during the learning block (dashed red lines), compared with color sensitivity calculated over all movement directions (solid lines) (same as Fig. $3 b$ ). Notation is as in Figure $3 b$. c $-\boldsymbol{e}$, Relations between neuronal responses and PDs. Dashed lines represent learning-related responses and color sensitivity recalculated for a specific subpopulation of the cells or behavioral trials. Solid lines represent learning-related responses and color sensitivity calculated for the entire neuronal population and all trials (same as Fig. $3 a, b)$. Notation is as in Figure $3, a$ and $b$. c, Learning-related responses (top) and color sensitivity (bottom), calculated separately for directionally tuned cells (solid line) versus nontuned cells (dashed lines) ( $n=47$ and 45 tuned cells, $n=93$ and 95 nontuned cells, for T0 and M0, respectively). $\boldsymbol{d}$, Learning-related responses (top) and color sensitivity (bottom), calculated specifically for tuned cells having a PD $\left(<22^{\circ}\right)$ close to directions used during the learning block ( $n=12$ and 11 , for T0 and M0, respectively). $\boldsymbol{e}$, Learning-related responses (top) and color sensitivity (bottom), calculated only for tuned cells ( $n=47$ and 45 for $\mathrm{TO}$ and $\mathrm{MO}$, respectively), only for movement directions away $\left(>22^{\circ}\right)$ from their PD.

different types of learning involved. The tasks used in these previous studies required manipulation of movement direction, a parameter that is well represented in motor cortices before learning. Therefore, the main observed effect was subtle and specific adjustments of the existing directional representations of specific cells. In contrast, the color-direction association task used in our study included novel information regarding target colors (that are not normally represented in the motor cortices). Therefore, changes are less specific and affect larger populations of neurons.

Our results are congruent with additional previous reports of the primary motor cortex representing parameters beyond movement dynamics and kinematics, such as serial order or movement context (Carpenter et al., 1999; Matsuzaka et al., 2007). In the ventral premotor cortex there have been reports of neurons discriminating sensory stimuli when such stimuli instructed movement (Romo et al., 2004). Also, during associative learning, there were reports of firing rate changes in this area (although not representing the task parameters) (Mitz et al., 1991). Furthermore, there is agreement with psychophysical studies showing that the motor system can use contextual information (for example, for rapid task switching) (Krouchev and Kalaska, 2003; Wada et al., 2003; Mistry and Contreras-Vidal, 2004).

Contextual responses have been reported in prefrontal and posterior parietal cortices during learning (Chen and Wise, 1995; Asaad et al., 2000; Assad, 2003). Top-down modulation from these areas may play a role in formation of the contextual responses reported here. Other possible candidates for the origin of 
learning-related changes in the motor cortices are the horizontal connections within a given region (Li et al., 2004; Stettler et al., 2002; Gilbert and Sigman, 2007). In M1, the horizontal connections affect a large population of neurons (Georgopoulos et al., 2007) and have been shown to be modulated in the adult brain during and after learning (Rioult-Pedotti et al., 1998, 2000; Sanes and Donoghue, 2000). Future studies might attempt to isolate the source of the large-scale plastic changes observed in this study.

\section{Color sensitivity}

Traces of the learning-related responses were maintained after learning through the appearance of novel color sensitivity. It is important to note that the term color sensitivity does not imply visual-like selectivity to colors. Rather, it involves selectivity to colors (features) that served to instruct movement (as opposed to colors that were never relevant for movement preparation). We suggest that color sensitivity is a maintenance of the learningrelated responses, because most $(\sim 80 \%)$ of the neurons that show color sensitivity after learning also show learning-related responses (Fig. $3 c$ ), and there is a correlation between each cell's learning-related response and its color sensitivity (Fig. $3 d, e$ ).

The appearance of color sensitivity after learning a color-direction task demonstrates the extent of the representational repertoire of the primary motor and premotor cortices: during the postlearning center-out task, color is no longer relevant, and correct performance requires responses to target direction alone. Yet, in parallel to the well documented direction representation of these motor cortices, they exhibited rapidly emerging responses to sensory features such as color, owing only to the involvement of these features in guiding behavior on the previous associative task. These responses suggest that the motor cortices represent general features instructing movement, although they are not related to the specifics of the required motor output. Furthermore, they show that such representations can appear rapidly during learning and can be maintained beyond their immediate relevance.

\section{Relation to motor memory}

What is the function of these novel responses? The learningrelated responses and color sensitivity are unlikely candidates as neural correlates for learning the specifics of the arbitrary associations used. First, the task required discriminating between the two associations; however, the neurons did not provide the information needed for such discrimination but, rather, only discriminated the associative task from the center-out task. Second, both responses were independent of movement direction and not specific to cells of a certain PD or to cells that have cosine-like tuning curves, whereas the arbitrary association task requires associating color to one specific movement direction. Third, they were maintained after learning, when associations were no longer relevant, although the monkeys' performance showed a clear switch to the center-out task (their behavior was indistinguishable from the center-out task preceding learning). To test this possibility directly, we assessed the correlation between the neuronal responses (learning-related responses and color sensitivity) in each session and task acquisition (the number of trials required to reach plateau) (see Materials and Methods) during that session. The neuronal responses were not correlated to acquisition during either TO or MO.

Continuation of the representation of a task after it is terminated might imply attentional modulation, and there have been reports of attentional modulation in the premotor cortex (Boussaoud, 2001; Binkofski et al., 2002; Rowe et al., 2002). Our results do not fit with attention in its strictest form, because color sensitivity is maintained throughout the postlearning block without diminishing, and has no visible effect on the postlearning performance of the center-out task (Fig. $1 c-e$ ). However, they are in line with increased saliency of stimuli after they gain behavioral relevance.

Another possible role of the maintained color sensitivity is in consolidation of the task into long-term memory. Previous studies further support this suggestion by showing, for example, that inhibition of protein synthesis in M1, or application of TMS to M1, interferes with improved retention on the following day (Muellbacher et al., 2002; Luft et al., 2004; Robertson et al., 2004; Richardson et al., 2006), and that synchronous TMS to M1 improved this retention (Bütefisch et al., 2004). Molecular studies showed long-term changes in M1 activity emerging after several days of training on a novel task (Rioult-Pedotti et al., 1998, 2000; Sanes and Donoghue, 2000; Kleim et al., 2004; Jackson et al., 2006). We tested this possibility by assessing the correlation between the neuronal responses (learning-related responses and color sensitivity) in each session and the improvement in performance on the following session (see Materials and Methods and supplemental Fig. 5, available at www.jneurosci.org as supplemental material). Color sensitivity was found to be well correlated to consolidation during both TO and MO. Therefore, we suggest that color-sensitive neurons might serve as part of the neuronal network involved in consolidation of the task into long term memory. Further studies, observing the same neurons over prolonged periods of time, might be useful in addressing this hypothesis.

Overall, the data presented here suggest that a large portion of motor cortical cells show, in parallel to their representation of movement direction, rapid task-dependent changes both during and after associative learning, including rapid emergence of novel responses to various sensory features, when such features are behaviorally relevant.

\section{References}

Asaad WF, Rainer G, Miller EK (2000) Task-specific neural activity in the primate prefrontal cortex. J Neurophysiol 84:451-459.

Assad JA (2003) Neural coding of behavioral relevance in parietal cortex. Curr Opin Neurobiol 13:194-197.

Binkofski F, Fink GR, Geyer S, Buccino G, Gruber O, Shah NJ, Taylor JG, Seitz RJ, Zilles K, Freund HJ (2002) Neural activity in human primary motor cortex areas $4 \mathrm{a}$ and $4 \mathrm{p}$ is modulated differentially by attention to action. J Neurophysiol 88:514-519.

Blake DT, Strata F, Churchland AK, Merzenich MM (2002) Neural correlates of instrumental learning in primary auditory cortex. Proc Natl Acad Sci U S A 99:10114-10119.

Boussaoud D (2001) Attention versus intention in the primate premotor cortex. Neuroimage 14:S40-S45.

Brashers-Krug T, Shadmehr R, Bizzi E (1996) Consolidation in human motor memory. Nature 382:252-255.

Bütefisch CM, Khurana V, Kopylev L, Cohen LG (2004) Enhancing encoding of a motor memory in the primary motor cortex by cortical stimulation. J Neurophysiol 91:2110-2116.

Carpenter AF, Georgopoulos AP, Pellizzer G (1999) Motor cortical encoding of serial order in a context-recall task. Science 283:1752-1757.

Chen LL, Wise SP (1995) Neuronal activity in the supplementary eye field during acquisition of conditional oculomotor associations. J Neurophysiol 73:1101-1121.

Costa RM, Cohen D, Nicolelis MAL (2004) Differential corticostriatal plasticity during fast and slow motor skill learning in mice. Curr Biol 14:1124-1134.

Crist RE, Li W, Gilbert CD (2001) Learning to see: experience and attention in primary visual cortex. Nat Neurosci 4:519-525.

Gandolfo F, Li C, Benda BJ, Schioppa CP, Bizzi E (2000) Cortical correlates 
of learning in monkeys adapting to a new dynamical environment. Proc Natl Acad Sci U S A 97:2259-2263.

Georgopoulos AP (2000) Neural aspects of cognitive motor control. Curr Opin Neurobiol 10:238-241.

Georgopoulos AP, Kettner RE, Schwartz AB (1988) Primate motor cortex and free arm movements to visual targets in three-dimensional space. II. Coding of the direction of movement by a neuronal population. J Neurosci 8:2928-2937.

Georgopoulos AP, Merchant H, Naselaris T, Amirikian B (2007) Mapping of the preferred direction in the motor cortex. Proc Natl Acad Sci U S A 104:11068-11072.

Gilbert CD, Sigman M (2007) Brain states: top-down influences in sensory processing. Neuron 54:677-696.

Gilbert CD, Sigman M, Crist RE (2001) The neural basis of perceptual learning. Neuron 31:681-697.

Hatsopoulos NG, Ojakangas CL, Paninski L, Donoghue JP (1998) Information about movement direction obtained from synchronous activity of motor cortical neurons. Proc Natl Acad Sci U S A 95:15706-15711.

Jackson A, Mavoori J, Fetz EE (2006) Long-term motor cortex plasticity induced by an electronic neural implant. Nature 444:56-60.

Kalaska JF, Scott SH, Cisek P, Sergio LE (1997) Cortical control of reaching movements. Curr Opin Neurobiol 7:849-859.

Karni A, Meyer G, Jezzard P, Adams MM, Turner R, Ungerleider LG (1995) Functional MRI evidence for adult motor cortex plasticity during motor skill learning. Nature 377:155-158.

Karni A, Meyer G, Rey-Hipolito C, Jezzard P, Adams MM, Turner R, Ungerleider LG (1998) The acquisition of skilled motor performance: fast and slow experience-driven changes in primary motor cortex. Proc Natl Acad Sci U S A 95:861-868.

Kleim JA, Hogg TM, VandenBerg PM, Cooper NR, Bruneau R, Remple M (2004) Cortical synaptogenesis and motor map reorganization occur during late, but not early, phase of motor skill learning. J Neurosci 24:628-633.

Krouchev NI, Kalaska JF (2003) Context-dependent anticipation of different task dynamics: rapid recall of appropriate motor skills using visual cues. J Neurophysiol 89:1165-1175.

Li CS, Padoa-Schioppa C, Bizzi E (2001) Neuronal correlates of motor performance and motor learning in the primary motor cortex of monkeys adapting to an external force field. Neuron 30:593-607.

Li W, Piëch V, Gilbert CD (2004) Perceptual learning and top-down influences in primary visual cortex. Nat Neurosci 7:651-657.

Luft AR, Buitrago MM, Ringer T, Dichgans J, Schulz JB (2004) Motor skill learning depends on protein synthesis in motor cortex after training. J Neurosci 24:6515-6520.

Matsuzaka Y, Picard N, Strick PL (2007) Skill representation in the primary motor cortex after long-term practice. J Neurophysiol 97:1819-1832.

Mistry S, Contreras-Vidal JL (2004) Learning multiple visuomotor transformations: adaptation and context-dependent recall. Mot Control 8:534-546.

Mitz AR, Godschalk M, Wise SP (1991) Learning-dependent neuronal activity in the premotor cortex: activity during the acquisition of conditional motor associations. J Neurosci 11:1855-1872.

Muellbacher W, Ziemann U, Wissel J, Dang N, Kofler M, Facchini S, Bo- roojerdi B, Poewe W, Hallett M (2002) Early consolidation in human primary motor cortex. Nature 415:640-644.

Padoa-Schioppa C, Li CS, Bizzi E (2004) Neuronal activity in the supplementary motor area of monkeys adapting to a new dynamic environment. J Neurophysiol 91:449-473.

Papoulis A (1984) Probability, random variables, and stochastic processes, Ed 2. New York: McGraw-Hill.

Paz R, Vaadia E (2004) Learning-induced improvement in encoding and decoding of specific movement directions by neurons in the primary motor cortex. PLoS Biol 2:E45.

Paz R, Boraud T, Natan C, Bergman H, Vaadia E (2003) Preparatory activity in motor cortex reflects learning of local visuomotor skills. Nat Neurosci 6:882-890.

Paz R, Wise SP, Vaadia E (2004) Viewing and doing: similar cortical mechanisms for perceptual and motor learning. Trends Neurosci 27:496-503.

Paz R, Natan C, Boraud T, Bergman H, Vaadia E (2005) Emerging patterns of neuronal responses in supplementary and primary motor areas during sensorimotor adaptation. J Neurosci 25:10941-10951.

Richardson AG, Overduin SA, Valero-Cabré A, Padoa-Schioppa C, PascualLeone A, Bizzi E, Press DZ (2006) Disruption of primary motor cortex before learning impairs memory of movement dynamics. J Neurosci 26:12466-12470.

Rioult-Pedotti MS, Friedman D, Hess G, Donoghue JP (1998) Strengthening of horizontal cortical connections following skill learning. Nat Neurosci 1:230-234.

Rioult-Pedotti MS, Friedman D, Donoghue JP (2000) Learning-induced LTP in neocortex. Science 290:533-536.

Robertson EM, Pascual-Leone A, Miall RC (2004) Current concepts in procedural consolidation. Nat Rev Neurosci 5:576-582.

Romo R, Hernández A, Zainos A (2004) Neuronal correlates of a perceptual decision in ventral premotor cortex. Neuron 41:165-173.

Rowe J, Friston K, Frackowiak R, Passingham R (2002) Attention to action: specific modulation of corticocortical interactions in humans. Neuroimage 17:988-998.

Sanes JN, Donoghue JP (2000) Plasticity and primary motor cortex. Annu Rev Neurosci 23:393-415.

Schoups A, Vogels R, Qian N, Orban G (2001) Practicing orientation identification improves orientation coding in V1 neurons. Nature 412:459-553.

Shadmehr R, Brashers-Krug T (1997) Functional stages in the formation of human long-term motor memory. J Neurosci 17:409-419.

Shadmehr R, Holcomb HH (1997) Neural correlates of motor memory consolidation. Nature 277:821-825.

Stettler DD, Das A, Bennett J, Gilbert CD (2002) Lateral connectivity and contextual interactions in macaque primary visual cortex. Neuron 36:739-750.

Wada Y, Kawabata Y, Kotosaka S, Yamamoto K, Kitazawa S, Kawato M (2003) Acquisition and contextual switching of multiple internal models for different viscous force fields. Neurosci Res 46:319-331.

Wise SP, Moody SL, Blomstrom KJ, Mitz AR (1998) Changes in motor cortical activity during visuomotor adaptation. Exp Brain Res 121:285-299.

Zach N, Kanarek N, Inbar D, Grinvald Y, Milestein T, Vaadia E (2005) Segregation between acquisition and long-term memory in sensorimotor learning. Eur J Neurosci 22:2357-2362. 